\title{
A non-randomized confirmatory trial of an expanded indication for endoscopic submucosal dissection for intestinal-type gastric cancer (cT1a): the Japan Clinical Oncology Group study (JCOG0607)
}

\author{
Noriaki Hasuike ${ }^{1} \cdot$ Hiroyuki Ono $^{2} \cdot$ Narikazu Boku $^{3} \cdot$ Junki Mizusawa $^{4} \cdot$ \\ Kohei Takizawa $^{2} \cdot$ Haruhiko Fukuda $^{4} \cdot$ Ichiro Oda $^{5} \cdot$ Hisashi Doyama $^{6} \cdot$ \\ Kazuhiro Kaneko $^{7}$ - Shinichiro Hori $^{8} \cdot$ Hiroyasu Iishi ${ }^{9}$ - Yukinori Kurokawa ${ }^{10}$. \\ Manabu Muto ${ }^{11}$ - Gastrointestinal Endoscopy Group of Japan Clinical Oncology Group \\ (JCOG-GIESG)
}

Received: 8 December 2016/Accepted: 7 February 2017/Published online: 21 February 2017

(C) The International Gastric Cancer Association and The Japanese Gastric Cancer Association 2017

\begin{abstract}
Background Endoscopic resection has been limited to intestinal-type gastric cancer (cT1a) with a low risk of lymph node metastasis (T1a $\leq 2 \mathrm{~cm}$, without ulcers). This single-arm confirmatory trial evaluated the efficacy and safety of endoscopic submucosal dissection (ESD) for $>2 \mathrm{~cm}$ ulcer-negative and $\leq 3 \mathrm{~cm}$ ulcer-positive intestinaltype gastric cancer (cT1a).

Methods The eligibility criteria included endoscopically diagnosed cT1a, a single primary intestinal-type gastric adenocarcinoma, an ulcer-negative lesion of any size or a $\leq 3 \mathrm{~cm}$ ulcer-positive lesion, cNOM0, and no prior treatment. If ESD resulted in noncurative resection, surgical resection was added. The primary endpoint was the 5-year overall survival (OS) (planned sample size was 470 , with a one-sided alpha level of $2.5 \%$ ). The threshold 5-year OS was $86.1 \%$.
\end{abstract}

K. Kaneko deceased on October 16, 2016.

Noriaki Hasuike

nhasuike11@gmail.com

1 Gastrointestinal Center and Institute of Minimally Invasive Endoscopic Care (iMEC), Sano Hospital, Kobe, 5-21 Shimizugaoka, Tarumi-Ku, Kobe 655-0031, Japan

2 Division of Endoscopy, Shizuoka Cancer Center, Shizuoka, Japan

3 Gastrointestinal Medical Oncology Division, National Cancer Center Hospital, Tokyo, Japan

4 Japan Clinical Oncology Group Data Center, National Cancer Center, Tokyo, Japan

5 Endoscopy Division, National Cancer Center Hospital, Tokyo, Japan
Results We enrolled 470 early gastric cancer patients [median tumor size, $25(5-130) \mathrm{mm}$ ] from 29 institutions between June 2007 and October 2010. These patients had 152 ulcer-negative lesions $(>2$ and $\leq 3 \mathrm{~cm}), 111$ ulcernegative lesions $(>3 \mathrm{~cm})$, and 207 ulcer-positive lesions $(\leq 3 \mathrm{~cm})$. The success rate for en block resection was 99.1\% (466/470). Additional gastrectomy was conducted in 131 patients (28\%) who did not fulfill the curative resection criteria. The 5-year OS of all patients was $97.0 \%$ (95\% confidence interval, 95.0-98.2\%), which was higher than the threshold 5-year OS $(86.1 \%)$. The 317 patients who satisfied the curative resection criteria had no recurrence. There were no ESD-related grade 4 adverse events.

Conclusion ESD for early gastric cancers that met the expanded criteria for intestinal-type gastric cancer (cT1a) was acceptable and should be the standard treatment instead of gastrectomy.

6 Department of Gastroenterology, Ishikawa Prefectural Central Hospital, Kanazawa, Ishikawa, Japan

7 Department of Gastroenterology, Endoscopy Division, National Cancer Center Hospital East, Kashiwa, Chiba, Japan

8 Department of Endoscopy, Shikoku Cancer Center, Matsuyama, Japan

9 Department of Gastrointestinal Oncology, Osaka Medical Center for Cancer and Cardiovascular Disease, Osaka, Japan

10 Department of Gastroenterological Surgery, Osaka University Graduate School of Medicine, Osaka, Japan

11 Department of Therapeutic Oncology, Kyoto University Graduate School of Medicine, Kyoto, Japan 
Keywords Endoscopy - Stomach neoplasms - Endoscopic submucosal resection · Gastrectomy

\section{Introduction}

Gastric cancer is one of the most common malignant diseases in the world. Clinical outcomes of gastric cancer have recently been improved due to early detection and curative resection. In Japan and Korea, the number of patients with $\mathrm{T} 1$ disease has increased since the establishment of a nationwide screening system; these patients now account for more than half of all gastric cancer patients. As early gastric cancer without lymph node and distant metastasis can be cured by limited local resection, endoscopic diagnostic and resection techniques have been rapidly developed [1-3]. However, considering that the clinical outcomes of endoscopic local resection should be comparable, not inferior, to those of surgical resection with lymph node dissection [4], endoscopic resection has been limited to patients with a very low risk of metastasis. In addition, since the pathological findings of resected specimens-such as histological differentiation, presence or absence of cancer cells at the cut end, and vessel invasion-are essential for estimating the risk of metastasis, en block resection is a very important technical issue when performing endoscopic resection [1, 5-8]. At present, the indication criteria for endoscopic resection of early gastric cancer recommended by the Japanese Gastric Cancer Association and the Japan Gastroenterological Endoscopy Society include (1) intestinal-type adenocarcinoma, (2) endoscopically diagnosed intramucosal cancer (cT1a), (3) tumor size $\leq 2 \mathrm{~cm}$, and (4) no endoscopic findings of ulcer (UL) $[1,9-11]$.

Recently, a new endoscopic resection technique, endoscopic submucosal dissection (ESD), was developed. This technique enables endoscopists to cut into the submucosal layer in an intended direction and remove the mucosal cancer, regardless of its size and whether a UL is present [12]. Thus, ESD may potentially solve the technical problems associated with conventional endoscopic resection.

Previous data from surgically resected specimens suggest that the risk of lymph node metastasis of cT1a gastric cancer is less than $1 \%$ if the following criteria are satisfied: (1) intestinal (differentiated)-type gastric adenocarcinoma which is UL-negative $>2 \mathrm{~cm}$ in size or which is UL-positive $\leq 3 \mathrm{~cm}$ in size or (2) diffuse (undifferentiated)-type gastric adenocarcinoma which is UL-negative and $\leq 2 \mathrm{~cm}$ in size $[6,7]$ This suggests that the indication for ESD can be expanded to these criteria for both intestinal and diffuse types of mucosal gastric adenocarcinoma. For intestinal- type gastric adenocarcinomas that satisfy the above criteria, the Japan Clinical Oncology Group (JCOG) conducted a multi-institution, single-arm, confirmatory trial (JCOG0607) to evaluate the efficacy and safety of ESD [13].

\section{Patients and methods}

\section{Trial design and settings}

This multi-institution, single-arm, confirmatory trial was conducted by the JCOG. The study protocol was reviewed and approved by the Protocol Review Committee of the JCOG and the institutional review board at each participating institution prior to initiation of the study. The study was conducted in accordance with the precepts established in the Declaration of Helsinki. Patients who were eligible for participation provided written informed consent before registration. This trial was registered with the University Hospital Medical Information Network Clinical Trials Registry (http://www.umin.ac.jp/ctr/index.htm), and the registration number was UMIN000000737.

\section{Inclusion and exclusion criteria}

The inclusion criteria were (1) histologically proven intestinal-type adenocarcinoma of the stomach by biopsy, (2) primary and single tumor, (3) endoscopically diagnosed as mucosal (cT1a) tumor, (4) UL-negative tumor $>2 \mathrm{~cm}$ in size or UL-positive tumor $\leq 3 \mathrm{~cm}$ in size, (5) predicted to be completely removed by en bloc resection with ESD, (6) low risk of severe stenosis after ESD, (7) no lymph node or distant metastasis ( $\mathrm{cN} 0$ and $\mathrm{cM} 0$ ) based on an abdominal computed tomography (CT) scan, (8) aged 20-75 years, (9) Eastern Cooperative Oncology Group performance status of 0 or 1 , (10) no prior gastrectomy or endoscopic treatment for simultaneous or metachronous gastric cancer and no reconstructive surgery using the stomach after resection of esophageal cancer, (11) no prior chemotherapy or radiation therapy against any other malignancies, (12) sufficient organ function, and (13) written informed consent. Exclusion criteria included (1) regular use of anticoagulant or antiplatelet medication, (2) simultaneous or metachronous (within 5 years) malignancy other than carcinoma in situ or mucosal cancer, (3) pregnant or breastfeeding women, (4) severe mental disease, (5) systemic administration of corticosteroids, (6) active bacterial or fungal infection, (7) concurrent unstable angina or myocardial infarction within 3 months before registration, (8) unstable hypertension, (9) uncontrolled or insulin-controlled diabetes mellitus, or (10) severe respiratory disease requiring continuous oxygen therapy. 


\section{Endpoints}

The primary endpoint was the 5-year overall survival (OS). OS was defined as the time from enrollment to death, irrespective of the cause, and it was censored at the last contact date for living patients. Secondary endpoints included recurrence-free survival (RFS), adverse events, and 5-year RFS with preserved stomach. RFS was defined as the time from registration to either the first event of recurrence or death from any cause, and it was censored at the last date when the patient was alive without recurrence. RFS with preserved stomach was defined as the time from registration to recurrence, gastrectomy, or death from any cause. Adverse events were evaluated according to the Common Terminology Criteria for Adverse Events version 3.0.

\section{Endoscopic submucosal dissection}

Lesion resection was performed using the ESD technique by an endoscopist certified as having performed ESD on at least 100 patients or by an experienced staff member under the supervision of a certified endoscopist. The routine procedure involved marking around the lesion, performing a circumferential mucosal incision outside the marked points, and performing submucosal dissection starting from the circumferential incision to remove the lesion [14]. The ESD device was selected by each endoscopist and included the IT Knife $^{\circledR}$ [15], Hook Knife ${ }^{\circledR}$, and Flex Knife ${ }^{\circledR}$, among others. Video recording was mandatory in all cases; some videos were reviewed at meetings of the study group to check that the technical quality of ESD was sufficiently high.

\section{Gross and pathologic evaluation}

Tumor size, location, and macroscopic types were endoscopically evaluated and classified by the Japanese Gastric Cancer Association Classification [16]. Intestinal (differentiated)-type gastric cancer was defined as papillary adenocarcinoma (pap), well-differentiated adenocarcinoma (tub1), or moderately differentiated adenocarcinoma (tub2). Diffuse (undifferentiated)-type gastric cancer was defined as a solid-type poorly differentiated adenocarcinoma (por1), non-solid-type poorly differentiated adenocarcinoma (por2), or signet-ring cell carcinoma. The depth of invasion, lymphatic and vascular involvement, and tumor involvement at the lateral and vertical margins were pathologically assessed.

\section{Assessment of curability}

Tumor removal in a single piece without macroscopically residual disease was defined as en bloc resection. En bloc resection showing lateral and vertical margins to be tumor- free on histological examination was defined as complete resection, and that showing cancer cells at the resection margin was defined as incomplete resection. Multiple fragment resection was also defined as incomplete resection, even if it resulted in tumor-free vertical margins with no macroscopic residual disease because the lateral margin could not be evaluated. Curative resection (CR) required the fulfillment of all of the following criteria on histological examination of the resected specimen: (1) intestinaltype adenocarcinoma, (2) pT1a(M) of any size or pT1b(SM1) (within $500 \mu \mathrm{m}$ from the lamina muscularis mucosae) if $\leq 3 \mathrm{~cm}$ in size, (3) tumor-free at the vertical margin, (4) UL-negative tumor of any size or UL-positive tumor $\leq 3 \mathrm{~cm}$ in size, and (5) no lymphatic or vascular involvement. Curability was classified, according to the resection mode, as complete curative resection (CCR) or incomplete curative resection (ICR). If none of the criteria for CR were satisfied, the case was classified as noncurative resection (NCR).

While no additional treatment was required for CCR cases, surgical resection was recommended for NCR cases. For ICR cases, surgical resection was not mandatory, and either additional ESD or observation without additional treatment was permitted.

\section{Complications}

When symptoms of melena, hematochezia, or hematemesis were noted after ESD, all bleeding events were confirmed by emergency endoscopy. Perforation was diagnosed by observation of (1) mesenteric fat on endoscopy or (2) free air on abdomen radiography or CT scan.

\section{Follow-up}

Esophagogastroduodenoscopy at 2 months after ESD was performed to confirm healing of the artificial ulcer and to check for residual tumors. During follow up, esophagogastroduodenoscopy (to check for local recurrence and metachronous tumor) and abdominal CT (to detect lymphnode or distant metastasis) were repeated at least once a year for 5 years.

\section{Statistical analysis}

This trial was designed as a confirmatory trial to determine the efficacy and safety of ESD for cT1aNOM0 gastric cancer in terms of 5-year OS. The primary endpoint was the 5-year OS for all enrolled patients. At the planning phase, the expected 5-year OS was tentatively set at $90 \%$ based on the age- and sex-adjusted 5-year OS calculated from the 1991-2000 population using the abridged life tables for 2004 in Japan [17]. Prior to the primary analysis, 
the expected 5-year OS was calculated based on the actual age and sex distribution of the enrolled patients, and the threshold 5-year OS was set at $85 \%$, a value 5\% lower than the expected 5-year OS. The initial planned sample size was 330 , with a one-sided alpha level of $5 \%$ and a power of $80 \%$. The protocol was amended to obtain a more precise estimate by increasing the sample size 1 year after the initiation of patient accrual because the patient accrual speed was better than expected. Thus, the sample size was increased from 330 to 470 patients with a one-sided alpha level of $2.5 \%$ and power of $90 \%$. The Kaplan-Meier method was used to estimate survival curves, and the confidence interval (CI) was estimated by Greenwood's formula. If the lower limit of the $95 \%$ CI for 5-year OS exceeded the threshold 5-year OS, the primary endpoint was met. Adverse events were assessed according to Common Terminology Criteria for Adverse Events version 3.0. The JCOG Data Center was responsible for data management, central monitoring, and statistical analysis. None of the physicians administering the interventions were involved in data analysis. All statistical analyses were performed with an intention-to-treat principle using SAS version 9.2 (SAS Institute, Cary, NC, USA).

\section{Results}

\section{Patients and clinicopathological characteristics}

A total of 470 patients with early gastric cancer (cT1aN0M0) were enrolled from 29 institutions between June 2007 and October 2010. There were no ineligible cases. The median age was 65 years (range $40-75$ years), and $85(18.1 \%)$ patients were female. The macroscopic appearance type was protruded in $155(33.0 \%)$, depressed in $258(54.9 \%)$, and mixed (combined protruded and depressed) in $57(12.1 \%)$ patients. A total of $71(15.1 \%)$, $255(54.3 \%)$, and $144(30.6 \%)$ lesions were located in the upper, middle, and lower third of the stomach, respectively. The median lesion size was $25 \mathrm{~mm}$ (range 5-130 mm). The tumor characteristics were UL-negative and $\leq 3 \mathrm{~cm}$ in size in $152(32.3 \%)$ patients, UL-negative and $>3 \mathrm{~cm}$ in size in $111(23.6 \%)$ patients, and UL-positive and $\leq 3 \mathrm{~cm}$ in size in $207(44.1 \%)$ patients (Table 1$)$.

\section{Endoscopic submucosal dissection results}

The median duration of ESD was 79 min (range 14-462 $\mathrm{min}$ ), and the median size of the resected tissue was $50 \mathrm{~mm}$ (range $21-122 \mathrm{~mm}$ ). Of 470 patients, 466 (99.1\%, 95\% CI, 97.8-99.8\%) underwent en bloc resection. In 3 patients, tumors were resected by multiple fragments, and ESD was discontinued because of perforation in one patient. Curability judged by resection mode and pathological findings was CCR in 317 (67\%) patients, ICR in $6(1 \%)$ patients, and NCR in $146(31 \%)$ patients (Table 2); 1 patient could not be evaluated because of emergent surgery for intraoperative perforation. Of the CCR patients, 34 patients had pT1a $\leq 2 \mathrm{~cm}$ and were ULnegative, 152 patients had pT1a $>2 \mathrm{~cm}$ and were ULnegative, 105 patients had pT1a $\leq 3 \mathrm{~cm}$ and were ULpositive, and 26 patients had pT1b (SM1 $<500 \mu \mathrm{m})$, $\leq 3 \mathrm{~cm}$.

The causes of NCR and ICR are shown in Table 3. There were multiple causes in some cases; a total of 268 causes (NCR 262, ICR 6) were reported in 152 patients (NCR 146, ICR 6). These causes were categorized as diagnostic, pathological, or both. Diagnostic causes were considered to be due to pre-ESD underdiagnosis, and pathological causes were revealed only with post-ESD pathological assessment. Among the reported 268 causes, $115(43 \%)$ were diagnostic (SM2 61, horizontal margin [+]

Table 1 Patient and tumor characteristics

\begin{tabular}{|c|c|c|}
\hline \multicolumn{3}{|l|}{ Age } \\
\hline $\operatorname{Min}-\max$ & $40-75$ & \\
\hline Median & 65 & \\
\hline \multicolumn{3}{|l|}{ Sex } \\
\hline Male & 385 & \\
\hline Female & 85 & \\
\hline Ulcerative findings and size & $\leq 3 \mathrm{~cm}$ & $>3 \mathrm{~cm}$ \\
\hline UL-negative & 152 & 111 \\
\hline UL-positive & 207 & 0 \\
\hline \multicolumn{3}{|l|}{ Tumor size $(\mathrm{mm})$} \\
\hline Min-max & $5-130$ & \\
\hline Median & 25 & \\
\hline \multicolumn{3}{|l|}{ Macroscopic type } \\
\hline Protruded type (0-I, 0-IIa) & 155 & \\
\hline Depressed type (0-IIb, 0-IIc, 0-III) & 258 & \\
\hline Mixed type $(0-\mathrm{III}+\mathrm{IIc}, 0$-IIc + IIa $)$ & 57 & \\
\hline \multicolumn{3}{|l|}{ Tumor location } \\
\hline Upper & 71 & \\
\hline Middle & 255 & \\
\hline Lower & 144 & \\
\hline \multicolumn{3}{|c|}{ Tumor location (transverse section) (multiple choice) } \\
\hline Lesser curvature & 231 & \\
\hline Greater curvature & 64 & \\
\hline Anterior wall & 89 & \\
\hline Posterior wall & 142 & \\
\hline \multicolumn{3}{|l|}{ Predominant histology } \\
\hline Pap & 6 & \\
\hline tub1 & 356 & \\
\hline tub2 & 108 & \\
\hline
\end{tabular}

UL ulcerative, Pap papillary adenocarcinoma, tubl well-differentiated adenocarcinoma, $t u b 2$ moderately differentiated adenocarcinoma 
or $[ \pm] 22$, vertical margin $[+]$ or $[ \pm] 32)$, and $81(30 \%)$ were pathological (dominantly undifferentiated-type 10, undifferentiated-type in SM invasion 20, lymphatic invasion 38, venous invasion 13), while 72 (27\%) were both (UL-positive $>3 \mathrm{~cm}: 44, \mathrm{pSM} 1>3 \mathrm{~cm}: 28$ ). A total of 132 patients, including 1 of 6 patients with ICR and 131 of 146 patients with NCR, underwent additional surgical resection (126 at the participating institutions and 6 at other hospitals), and 15 patients with NCR were followed up without additional treatment (Fig. 1). In the 132 patients with additional surgical resection, lymph node metastasis was pathologically detected in $7(5 \%)$.

\section{Complications}

Perforation occurred in 12 patients (grade 2 and 3: 2.6\%) during ESD (Table 4). Endoscopic closure by clipping was successful in 11 of these patients while emergent surgery was performed in 1 patient. Twenty-nine patients (grade 2 and 3: 6.2\%) required endoscopic hemostasis after ESD (within 30 days), and 3 of those patients received a blood transfusion. Post-ESD secondary hemorrhage tended to be observed more frequently in patients with a resected tissue size $>30 \mathrm{~mm}$ than in those with a resected tissue size $\leq 30 \mathrm{~mm} \quad(10.9 \%$ vs. $4.7 \%$, respectively, two-sided $P=0.024$ by Fisher's exact test). While grade 3 or 4 adverse events were observed in $7(5.3 \%)$ of 133 patients receiving additional surgical resection, grade 4 adverse events occurred in only 2 patients (acute pancreatitis and acute respiratory distress syndrome in 1 patient, central nervous system ischemia in 1 patient), both of whom recovered within a short period.

\section{Clinical outcomes}

The expected 5-year OS based on the actual age and sex distribution of enrolled patients was calculated as $91.1 \%$. Thus, the threshold 5-year OS was found to be $86.1 \%$. The number of patients lost to follow-up within 5 years was 22 (4.7\%). With a median follow-up of 73.8 months (range 9.3-98.8) for all enrolled patients, the 5-year OS was 97.0\% (95\% CI, 95.0-98.2\%) (Fig. 2a). The lower limit of the $95 \%$ CI of the 5-year OS was higher than the threshold 5-year OS $(86.1 \%)$, and the primary endpoint was met. The 5-year RFS in all enrolled patients was 96.9\% (95\% CI 94.9-98.2\%) (Fig. 2b). Among the 317 (67.4\%) patients followed up after achieving CCR, no local or distant recurrence was observed and no deaths due to gastric cancer were encountered. In contrast, among the 146 $(31.1 \%)$ patients with NCR, $3(0.6 \%)$ patients (pSM2 depth of invasion after ESD in all 3 patients) developed a recurrence (liver only in 1 patient; left adrenal gland only in 1 patient; and lymph node, liver, lung, and bone in 1
Table 2 Operation details and pathological results of endoscopic submucosal dissection $(n=470)$

\begin{tabular}{|c|c|}
\hline \multicolumn{2}{|l|}{ Operation duration (minutes) } \\
\hline Min-max & $14-462$ \\
\hline Median & 79 \\
\hline \multicolumn{2}{|l|}{ ESD device } \\
\hline IT knife, IT knife2 & 387 \\
\hline Hook knife & 31 \\
\hline Flex knife & 12 \\
\hline Needle knife & 8 \\
\hline Flush knife & 26 \\
\hline Other & 6 \\
\hline \multicolumn{2}{|l|}{ Size of resected specimen $(\mathrm{mm})^{\mathrm{a}}$} \\
\hline Median & 50 \\
\hline Min-max & $21-122$ \\
\hline \multicolumn{2}{|l|}{ Technical assessment $^{\mathrm{a}}$} \\
\hline Complete resection (en bloc) & 431 \\
\hline Incomplete resection (cut into the lesion) & 35 \\
\hline Incomplete resection (multiple fragments) & 3 \\
\hline \multicolumn{2}{|l|}{ Predominant type ${ }^{\mathrm{a}}$} \\
\hline Pap & 9 \\
\hline tub1 & 322 \\
\hline tub2 & 128 \\
\hline por1 & 5 \\
\hline por2 & 3 \\
\hline Sig & 2 \\
\hline \multicolumn{2}{|l|}{ Depth of invasion ${ }^{\mathrm{a}}$} \\
\hline $\mathrm{pM}$ & 346 \\
\hline pSM1 & 62 \\
\hline pSM2 & 59 \\
\hline SM invasive part undiff absent & 102 \\
\hline SM invasive part undiff present & 20 \\
\hline \multicolumn{2}{|l|}{ Ulcerative change $^{\mathrm{a}}$} \\
\hline UL-negative & 273 \\
\hline UL-positive & 196 \\
\hline \multicolumn{2}{|l|}{ Tumor size $(\mathrm{cm})^{\mathrm{a}}$} \\
\hline Median & 2.8 \\
\hline Min-max & $0.2-8.5$ \\
\hline \multicolumn{2}{|l|}{ Lymphatic involvement $^{\mathrm{a}}$} \\
\hline ly0 & 431 \\
\hline ly1 & 32 \\
\hline ly2 & 6 \\
\hline \multicolumn{2}{|l|}{ Vessel involvement $^{\mathrm{a}}$} \\
\hline v0 & 456 \\
\hline v1 & 12 \\
\hline v2 & 1 \\
\hline \multicolumn{2}{|l|}{ Horizontal margin $^{\mathrm{a}}$} \\
\hline Negative & 447 \\
\hline Positive & 9 \\
\hline Unknown & 13 \\
\hline Vertical margin $^{\mathrm{a}}$ & \\
\hline
\end{tabular}




$\begin{array}{ll}\text { Negative } & 437 \\ \text { Positive } & 13 \\ \text { Unknown } & 19 \\ \text { Curative assessment }^{\mathrm{a}} & \\ \text { Complete curative resection } & 317 \\ \text { Noncurative resection } & 146 \\ \text { Incomplete resection } & 6\end{array}$

$E S D$ endoscopic submucosal dissection, pap papillary adenocarcinoma, tubl well-differentiated adenocarcinoma, tub2 moderately differentiated adenocarcinoma, porl solid type of poorly differentiated adenocarcinoma, por 2 nonsolid type of poorly differentiated adenocarcinoma, sig signet-ring cell carcinoma, $U L$ ulcerative

a One patient who underwent emergency surgery is missing from the data

patient) after additional surgical resection. These 3 patients subsequently received systemic chemotherapy, but all died of gastric cancer. In addition, the 5-year RFS with preserved stomach was $68.8 \%$ (95\% CI $64.4-72.8 \%)$ in all patients and $95.8 \%$ (95\% CI 92.9-97.5\%) in those with CCR (Fig. 3a, b). Nine patients underwent gastrectomy for metachronous gastric cancer, and 1 patient underwent gastrectomy for a gastrointestinal stromal tumor after CCR. The 6 patients with ICR had no recurrence.

\section{Discussion}

This was the first multicenter confirmatory clinical trial evaluating the safety and efficacy of ESD for the expanded indication of intestinal-type gastric cancer (cT1a) including UL-negative tumor $>2 \mathrm{~cm}$ in size or UL-positive tumor $\leq 3$ $\mathrm{cm}$ in size. This trial demonstrated a good safety profile and good clinical outcomes in 470 patients with cT1aN0M0 for intestinal-type gastric adenocarcinoma. Complications associated with ESD were manageable, and the obtained lower limit of the 95\% CI of the 5-year OS clearly exceeded the prespecified threshold. In this analysis, we used the survival rate of the general population adjusted for sex and age as a reference to determine the expected and threshold values of the 5-year OS. However, this study was biased as patients with preserved organ function and without severe complications were enrolled due to the eligibility criteria, resulting in a lower frequency

Table 3 Causes of noncurative resection and incomplete resection (there were multiple causes in some cases)

\begin{tabular}{|c|c|c|c|c|c|c|c|c|c|}
\hline & \multicolumn{3}{|c|}{ Diagnostic } & \multicolumn{4}{|l|}{ Pathological } & \multicolumn{2}{|l|}{ Size } \\
\hline & SM2 & $\mathrm{HM}(+)$ or $( \pm)$ & $\mathrm{VM}(+)$ or $( \pm)$ & $\begin{array}{l}\text { Dominant undiff } \\
\text { type }\end{array}$ & Undiff type in SM invasion & ly+ & $\mathrm{v}+$ & $\begin{array}{l}\text { UL- } \\
\text { positive } \\
>3 \mathrm{~cm}\end{array}$ & $\begin{array}{l}\mathrm{pSM} 1 \\
>3 \mathrm{~cm}\end{array}$ \\
\hline $\begin{array}{l}\text { UL- } \\
\text { negative } \\
>2 \mathrm{~cm}\end{array}$ & 34 & 13 & 19 & 5 & 12 & 25 & 6 & 21 & 25 \\
\hline $\begin{array}{l}\text { UL-positive } \\
\leq 3 \mathrm{~cm}\end{array}$ & 27 & 9 & 13 & 5 & 8 & 13 & 7 & 23 & 3 \\
\hline Total & $\begin{array}{r}61 \\
115\end{array}$ & 22 & 32 & $\begin{array}{l}10 \\
81\end{array}$ & 20 & 38 & 13 & $\begin{array}{l}44 \\
72\end{array}$ & 28 \\
\hline
\end{tabular}

$H M$ horizontal margin, $S M$ submucosal, $U L$ ulcerative

Fig. 1 Patient flow diagram. $E S D$ endoscopic submucosal dissection, $C C R$ complete curative resection, ICR incomplete curative resection, $N C R$ noncurative resection

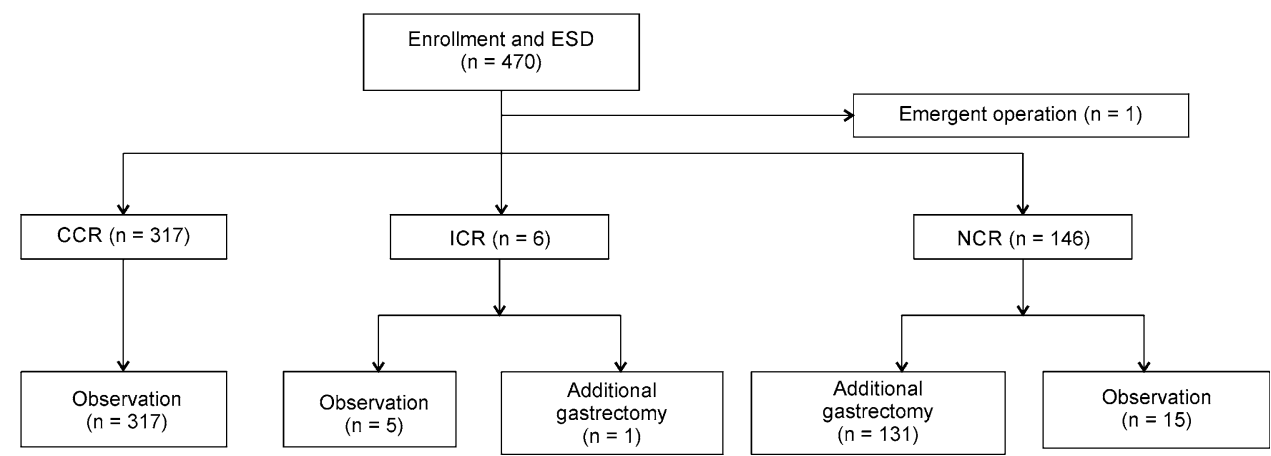


Table 4 Adverse events after endoscopic submucosal dissection

\begin{tabular}{lrrrrlllll}
\hline & $\mathrm{G} 0$ & $\mathrm{G} 1$ & $\mathrm{G} 2$ & $\mathrm{G} 3$ & $\mathrm{G} 4$ & $\% \mathrm{G} 2-4$ & $\% \mathrm{G} 3-4$ & $\% \mathrm{G} 4$ & Total \\
\hline Fever $\left(>38^{\circ} \mathrm{C}\right)$ & 437 & 30 & 2 & 0 & 0 & 0.4 & 0 & 0 & 469 \\
Aspiration & 466 & 1 & 1 & 1 & 0 & 0.4 & 0.2 & 0 & 469 \\
Appetite loss & 457 & 11 & 1 & 0 & 0 & 0.2 & 0 & 0 & 469 \\
Dysphagia & 469 & 0 & 0 & 0 & 0 & 0 & 0 & 0 & 469 \\
Heartburn & 458 & 11 & 0 & 0 & - & 0 & 0 & - & 469 \\
Nausea & 454 & 14 & 1 & 0 & 0 & 0.2 & 0 & 0 & 469 \\
Vomiting & 461 & 6 & 2 & 0 & 0 & 0.4 & 0 & 0 & 469 \\
Perforation: stomach & 458 & 0 & 11 & 1 & 0 & 2.6 & 0.2 & 0 & 470 \\
Pain: epigastric & 417 & 43 & 9 & 0 & 0 & 1.9 & 0 & 0 & 469 \\
Pain: throat & 458 & 11 & 0 & 0 & 0 & 0 & 0 & 0 & 469 \\
After bleeding: stomach & 429 & 11 & 26 & 3 & 0 & 6.2 & 0.6 & 0 & 469 \\
\hline
\end{tabular}

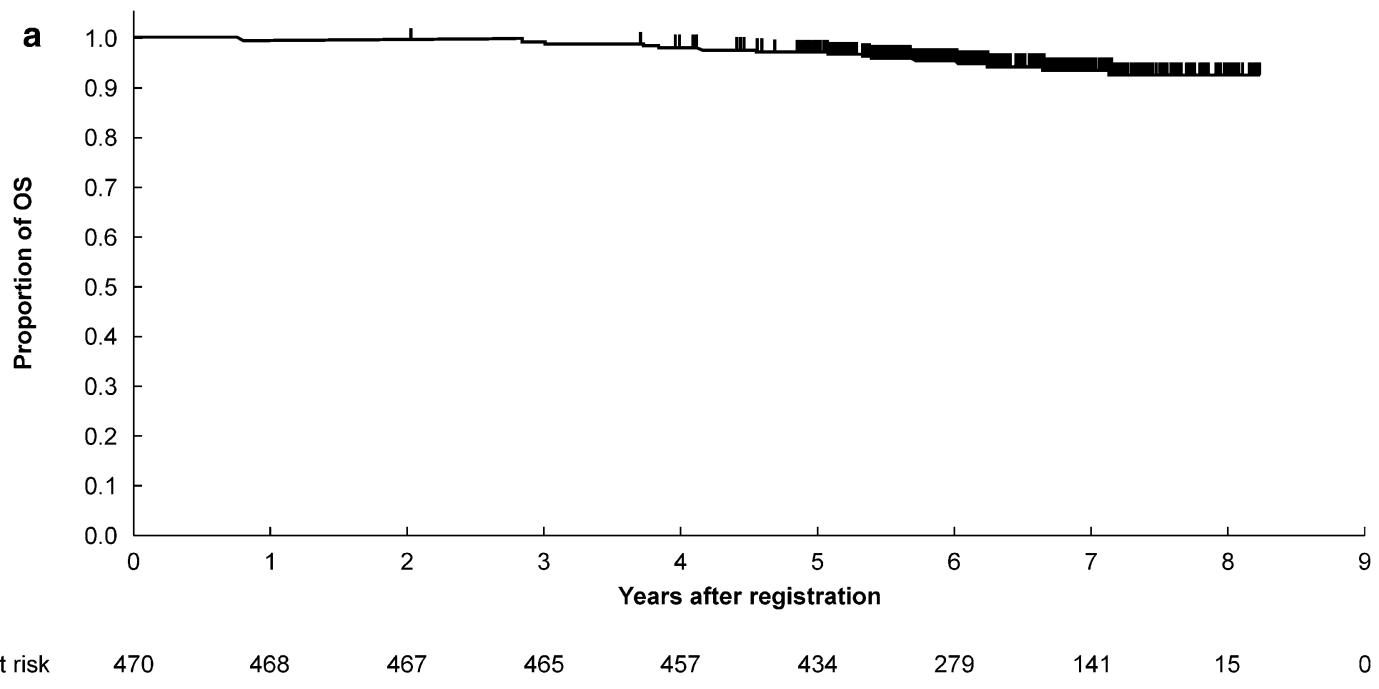

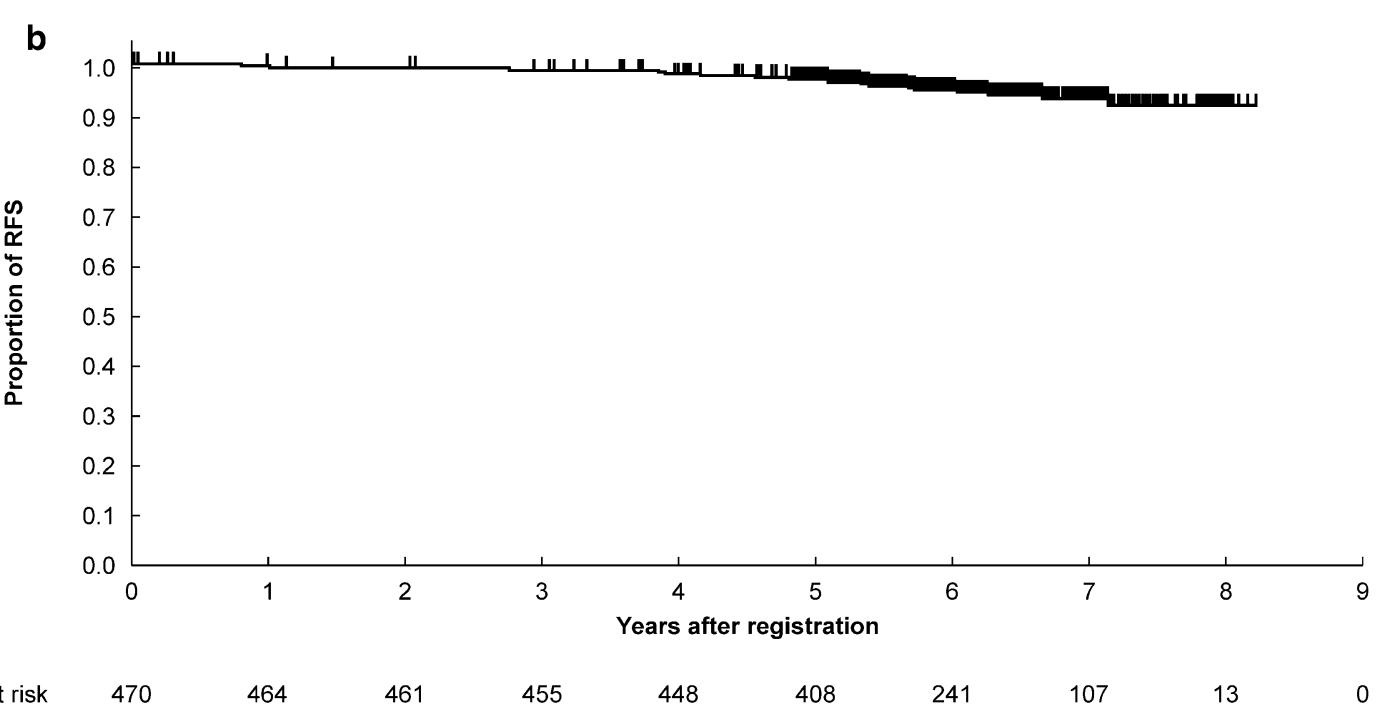

Fig. 2a-b Overall (a) and recurrence-free (b) survival for all enrolled patients. OS overall survival, RFS recurrence-free survival

of death due to other diseases compared to the general population. Another major limitation of this study is that it was not a randomized controlled trial. During the planning of this study, we expected it to be difficult to conduct a randomized phase III trial comparing surgical resection and ESD, which was very popular in Japan, so we designed this 

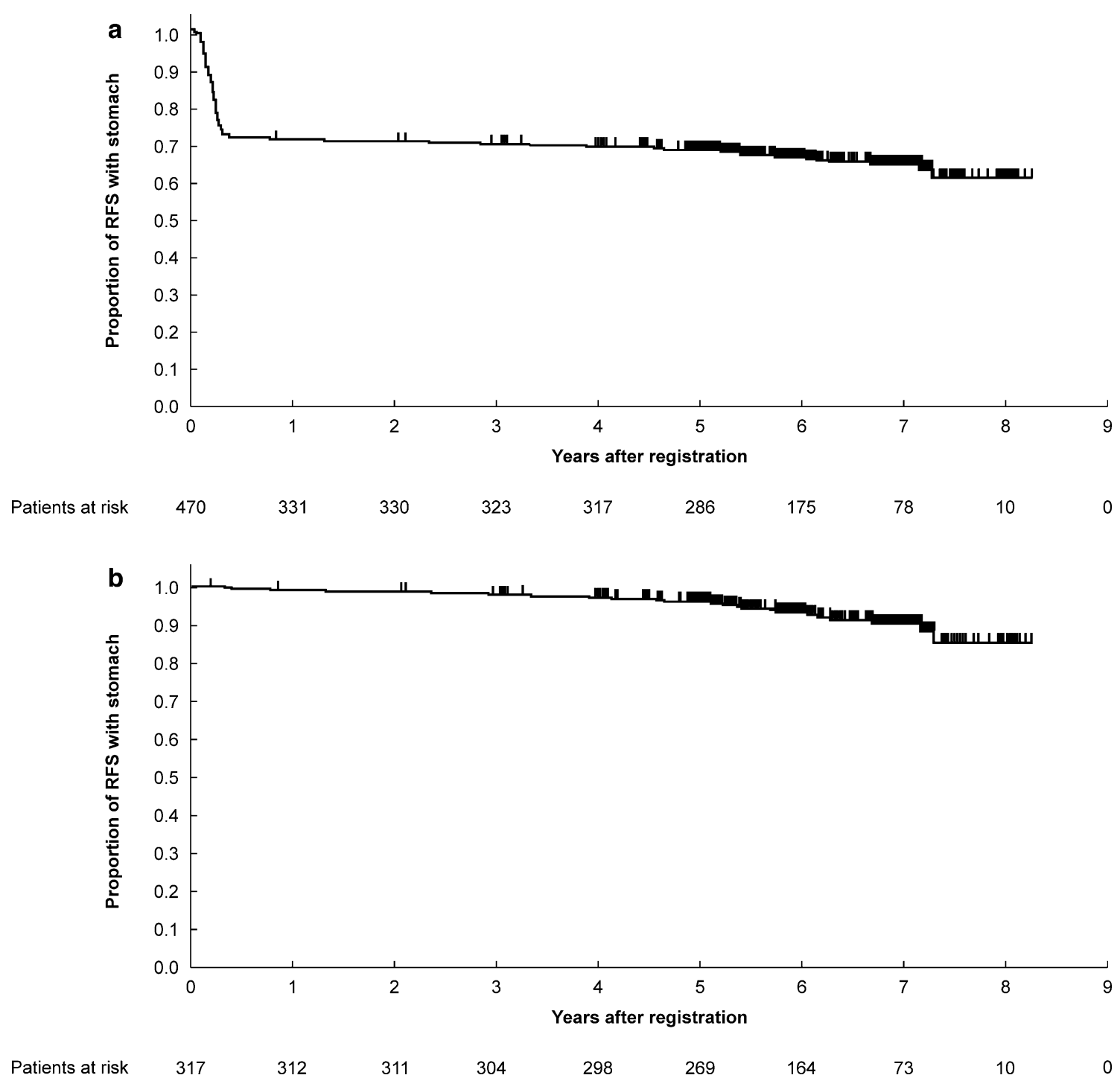

Fig. 3a-b Recurrence-free survival with preserved stomach for all enrolled patients (a) and complete curative resection patients (b). RFS recurrence-free survival

study as a confirmatory trial with a large sample size. However, there was no recurrence (100\% curability) in 317 and 6 patients with CCR and ICR, respectively, and all but 3 of the 146 patients with NCR could be salvaged by performing additional surgery. While the 5-year RFS with preserved stomach for all cases was $68.8 \%$, that for the patients with CCR was as high as $95.8 \%$. Thus, the expanded indication for ESD can be accepted without a randomized controlled trial, given that it markedly improved the patients' quality of life. However, metachronous gastric cancer in the preserved stomach is another problem. In this study, 9 patients underwent gastrectomy for metachronous gastric cancer. The appropriate interval between endoscopic examinations needed to ensure that the patient's stomach is preserved throughout their life should be investigated in future trials.
One reason for the excellent outcomes obtained in our study was the endoscopists' technique in Japan. En bloc resection, which was obtained in $99.1 \%$ of the subjects, enables precise pathological evaluation. When planning this study, we believed that the ESD technique would be very important, as previous reports indicated an increased perioperative risk of complications and poor clinical outcomes due to lack of experience $[6,8,8,18-20]$. In this study, only certified endoscopists that had performed more than 100 ESDs were allowed to perform ESD [3, 21, 22]. Endoscopist training for ESD is necessary to generalize the results of this study worldwide.

Another reason for the excellent outcomes attained in this study was the strict criteria applied for curative resection. The criteria for evaluating curability were determined based on previous reports of the frequency of lymph node 
metastasis pathologically detected after gastrectomy, which suggested that CCR was associated with nearly $0 \%$ possibility of lymph node metastasis [6]. In other words, patients who did not satisfy the criteria for CCR had some risk of metastasis. Indeed, 3 patients (2\%) with NCR died of gastric cancer after a recurrence with distant metastasis, even after additional surgical resection. Nevertheless, the outcome of additional surgery in this study was better than the previously reported 5-year OS of $89.3 \%$ for stage Ib gastric cancer $[1,3,17,23]$. The good outcomes obtained after additional surgery partly contributed to the overall results of this study. However, the risk of lymph node metastasis in patients with NCR was estimated to be less than $10 \%$ in previous reports of surgical resection [24]. In fact, lymph node metastasis was detected in only $5 \%$ of the 131 patients with additional surgical resection. The present criteria for additional surgical resection after NCR may be too strict from a quality of life viewpoint because additional surgery would be considered overtreatment for more than $90 \%$ of patients without lymph node metastasis. New biomarkers predicting individual metastasis risk should be developed, in addition to pathological findings, to expand the indication for ESD and preserve the stomach in more patients.

Although CCR was attained in $67 \%$ of patients, in most of whom the stomach could be preserved, additional surgical gastrectomy was performed in $28 \%$ of the patients for whom ESD was not considered to be necessary. Among the causes of additional surgical resection, diagnostic causesaccounting for $43 \%$ of those patients-could be prevented by more accurate endoscopic diagnosis. In this study, each endoscopist decided which diagnostic tools to use, such as endoscopic ultrasound sonography. Generally, the accuracy rate when differentiating between mucosal and submucosal gastric cancer has been reported to be approximately $80 \%$ [25-28]. The relatively high rate of underdiagnosis in this study was likely due to the study population, as the extended indication criteria for ESD (such as large size and presence of UL) made depth diagnosis difficult. In the future, endoscopic diagnosis for tumor depth and spread should be improved by applying new image-enhanced endoscopy techniques such as narrow-band imaging.

As for the adverse events associated with ESD, Oka et al. noted that the incidence of perforation with ESD was significantly higher (53.8\%) in cases of UL and advocated special measures for ESD of UL-positive lesions [4]. However, the overall incidence of perforation was as low as $2.6 \%$ in our study. This can be attributed to the fact that, in our expanded indication criteria, we limited the size of UL-positive lesions to $\leq 3 \mathrm{~cm}$. This confirmatory trial indicates that the expanded indication criteria for ESD of intestinal-type gastric cancer (cT1a) do not notably increase the perforation risk $[4,21,22,29,30]$. Isomoto et al. [31] reported a perforation incidence of $4.6 \%$, and that bleeding requiring a blood transfusion occurred in only a small percentage of patients. In this study, $6.2 \%$ of the patients required endoscopic hemostasis within 30 days after ESD, and 3 of these patients received a blood transfusion. These results highlight the good safety profile of the expanded ESD indication for intestinal-type gastric cancer (cT1a). However, it should be recognized that postoperative secondary hemorrhage tended to be more common in patients with a resected tissue size $>3 \mathrm{~cm}$.

In conclusion, this confirmatory clinical trial indicates that ESD for early gastric cancer that satisfies the expanded criteria of intestinal-type gastric cancer (cT1a) and ULnegative tumor $>2 \mathrm{~cm}$ in size or UL-positive tumor $\leq 3 \mathrm{~cm}$ in size showed a 5-year OS of $97.0 \%$ and was associated with few adverse events, all of which were manageable. Furthermore, the 5-year RFS with preserved stomach was $68.8 \%$. ESD is recommended for early gastric cancer that complies with the expanded criteria for intestinal-type gastric cancer (cT1a) instead of surgical gastrectomy.

Acknowledgements This study was supported by Grants-in-Aid for Cancer Research (17S-3, 17S-5, 20S-3, 20S-6, 23-A-16, 23-A-19, 26-A-4) and Health and Labour Sciences Research Grants for Clinical Cancer Research (17-12, 20-15) from the Ministry of Health, Labour and Welfare, Japan. Participating institutions: Japan Clinical Oncology Group (JCOG); Gastrointestinal Endoscopy Study Group; Hokkaido University Hospital; Federation of National Public Service Personnel Mutual Aid Associations, Tonan Hospital; Tokyo Metropolitan Cancer and Infectious Diseases Center Komagome Hospital; Asahi General Hospital; Ishikawa Prefectural Central Hospital; Saku Central Hospital Advanced Care Center; Shizuoka Cancer Center; Aichi Cancer Center Hospital; Aichi Cancer Center, Aichi Hospital; Kyoto University Hospital; Osaka Prefectural Hospital Organization, Osaka Medical Center for Cancer and Cardiovascular Diseases; Osaka City General Hospital; Kobe University Graduate School of Medicine; Hyogo Cancer Center; National Hospital Organization Shikoku Cancer Center; Kochi Health Sciences Center; Tochigi Cancer Center; Yamagata Prefectural Central Hospital; Ibaraki Prefectural Central Hospital \& Cancer Center; Showa University School of Medicine; Cancer Institute Hospital of Japanese Foundation for Cancer Research; Toranomon Hospital; Yokohama City University Medical Center; Kanagawa Cancer Center; Kitasato University School of Medicine; National Cancer Center Hospital East; Chiba Cancer Center; National Cancer Center Hospital; Iwate Prefectural Central Hospital.

\section{Compliance with ethical standards}

Human rights statement and informed consent All procedures followed were in accordance with the ethical standards of the responsible committee on human experimentation (institutional and national) and with the Helsinki Declaration of 1964 and later versions. The study protocol was reviewed and approved by the Protocol Review Committee of JCOG and the institutional review board at each participating institution prior to initiation of the study. Written informed consent was obtained from all patients before they were included in the study.

Conflict of interest The authors declare that they have no conflict of interest. 


\section{References}

1. Gotoda T. Endoscopic resection of early gastric cancer. Gastric Cancer. 2007;10:1-11.

2. Oda I, Gotoda T, Hamanaka H, Eguchi T, Saito Y, Matsuda T, et al. Endoscopic submucosal dissection for early gastric cancer: technical feasibility, operation time and complications from a large consecutive series. Dig Endosc. 2005;17:54-8.

3. Gotoda T, Kusano C, Moriyasu F. Future perspective of gastric cancer endotherapy. Ann Transl Med. 2014;2:25.

4. Oka S, Tanaka S, Kaneko I, Mouri R, Hirata M, Kawamura T, et al. Advantage of endoscopic submucosal dissection compared with EMR for early gastric cancer. Gastrointest Endosc. 2006;4:877-83.

5. Ohta H, Noguchi Y, Takagi K, Nishi M, Kajitani T, Kato Y. Early gastric carcinoma with special reference to macroscopic classification. Cancer. 1987;60:1099-106.

6. Gotoda T, Yanagisawa A, Sasako M, Ono H, Nakanishi Y, Shimoda $\mathrm{T}$, et al. Incidence of lymph node metastasis from early gastric cancer: estimation with a large number of cases at two large centers. Gastric Cancer. 2000;3:219-25.

7. Hirasawa T, Gotoda T, Miyata S, Kato Y, Shimoda T, Taniguchi $\mathrm{H}$, et al. Incidence of lymph node metastasis and the feasibility of endoscopic resection for undifferentiated-type early gastric cancer. Gastric Cancer. 2009;12:148-52.

8. Sano T, Kobori O, Muto T. Lymph node metastasis from early gastric cancer: endoscopic resection of tumour. $\mathrm{Br} \mathrm{J}$ Surg. 1992;79:241-4.

9. Japanese Gastric Cancer Association. Japanese classification of gastric carcinoma. 3rd English edition. Gastric Cancer. 2011;14:101-12.

10. Association Japanese Gastric Cancer. Japanese gastric cancer treatment guidelines 2010 (ver. 3). Gastric Cancer. 2011;14:113-23.

11. Ono H, Yao K, Fujishiro M, Oda I, Nimura S, Yahagi N, et al. Guidelines for endoscopic submucosal dissection and endoscopic mucosal resection for early gastric cancer. Dig Endosc. 2016;28:3-15.

12. Ono H, Kondo H, Gotoda T, Shirao K, Yamaguchi H, Saito D, et al. Endoscopic mucosal resection for treatment of early gastric cancer. Gut. 2001;48:225-9.

13. Kurokawa Y, Hasuike N, Ono H, Boku N. Fukuda H; Gastrointestinal Oncology Study Group of Japan Clinical Oncology Group. A phase II trial of endoscopic submucosal dissection for mucosal gastric cancer: Japan Clinical Oncology Group Study JCOG0607. Jpn J Clin Oncol. 2009;39:464-6.

14. Conlin A, Kaltenbach T, Kusano C, Matsuda T, Oda I, Gotoda T. Endoscopic resection of gastrointestinal lesions: advancement in the application of endoscopic submucosal dissection. J Gastroenterol Hepatol. 2010;25:1348-57.

15. Ono H, Hasuike N, Inui T, Takizawa K, Ikehara H, Yamaguchi $\mathrm{Y}$, et al. Usefulness of a novel electrosurgical knife, the insulation-tipped diathermic knife-2, for endoscopic submucosal dissection of early gastric cancer. Gastric Cancer. 2008;11:47-52.

16. Park CH, Shin S, Park JC, Shin SK, Lee SK, Lee YC, et al. Longterm outcome of early gastric cancer after endoscopic submucosal dissection: expanded indication is comparable to absolute indication. Dig Liver Dis. 2013;45:651-6.
17. Ministry of Health, Labour, and Welfare. Abridged life table 2004 (online). Tokyo: Ministry of Health, Labour, and Welfare, 2005. http://www.mhlw.go.jp/toukei/saikin/hw/life/ life04/. Accessed 16 Sep 2016.

18. Gotoda T, Yamamoto H, Soetikno RM. Endoscopic submucosal dissection of early gastric cancer. J Gastroenterol. 2006;41:929-42.

19. Sano T, Sasako M, Kinoshita T, Maruyama K. Recurrence of early gastric cancer. Follow-up of 1475 patients and review of the Japanese literature. Cancer. 1993;72:3174-8.

20. Ludwig K, Klautke G, Bernhard J, Weiner R. Minimally invasive and local treatment for mucosal early gastric cancer. Surg Endosc. 2005;19:1362-6.

21. Oda I, Odagaki T, Suzuki H, Nonaka S, Yoshinaga S. Learning curve for endoscopic submucosal dissection of early gastric cancer based on trainee experience. Dig Endosc. 2012;24(Suppl 1):129-32.

22. Kakushima N, Fujishiro M, Kodashima S, Muraki Y, Tateishi A, Omata M. A learning curve for endoscopic submucosal dissection of gastric epithelial neoplasms. Endoscopy. 2006;38:991-5.

23. Hirasawa K, Kokawa A, Oka H, Yahara S, Sasaki T, Nozawa A, et al. Risk assessment chart for curability of early gastric cancer with endoscopic submucosal dissection. Gastrointest Endosc. 2011;74:1268-75.

24. Choi JY, Park YS, Jung HY, da Son H, Ahn JY, Han S, et al. Identifying predictors of lymph node metastasis after endoscopic resection in patients with minute submucosal cancer of the stomach. Surg Endosc. 2015;29:1476-83.

25. Sano T, Okuyama Y, Kobori O, Shimizu T, Morioka Y. Early gastric cancer. Endoscopic diagnosis of depth of invasion. Dig Dis Sci. 1990;35:1340-4.

26. Yanai H, Noguchi T, Mizumachi S, Tokiyama H, Nakamura H, Tada M, et al. A blind comparison of the effectiveness of endoscopic ultrasonography and endoscopy in staging early gastric cancer. Gut. 1999;44:361-5.

27. Choi J, Kim SG, Im JP, Kim JS, Jung HC, Song IS. Endoscopic prediction of tumor invasion depth in early gastric cancer. Gastrointest Endosc. 2011;73:917-27.

28. Seto Y, Shimoyama S, Kitayama J, Mafune K, Kaminishi M, Aikou T, et al. Lymph node metastasis and preoperative diagnosis of depth of invasion in early gastric cancer. Gastric Cancer. 2001;4:34-8.

29. Fujishiro M, Yahagi N, Nakamura M, Kakushima N, Kodashima $\mathrm{S}$, Ono $\mathrm{S}$, et al. Successful outcomes of a novel endoscopic treatment for GI tumors: endoscopic submucosal dissection with a mixture of high-molecular-weight hyaluronic acid, glycerin, and sugar. Gastrointest Endosc. 2006;63:243-9.

30. Nakamoto S, Sakai Y, Kasanuki J, Kondo F, Ooka Y, Kato K, et al. Indications for the use of endoscopic mucosal resection for early gastric cancer in Japan: a comparative study with endoscopic submucosal dissection. Endoscopy. 2009;41:746-50.

31. Isomoto H, Shikuwa S, Yamaguchi N, Fukuda E, Ikeda K, Nishiyama H, et al. Endoscopic submucosal dissection for early gastric cancer: a large-scale feasibility study. Gut. 2009;58:331-6. 